\title{
Multiplasmon Absorption in Graphene
}

\author{
Marinko Jablan ${ }^{1,2, *}$ and Darrick E. Chang ${ }^{1}$ \\ ${ }^{1}$ ICFO-Institut de Ciencies Fotoniques, Mediterranean Technology Park, 08860 Castelldefels (Barcelona), Spain \\ ${ }^{2}$ Department of Physics, University of Zagreb, 10000 Zagreb, Croatia
}

(Received 21 January 2015; published 10 June 2015)

\begin{abstract}
We show that graphene possesses a strong nonlinear optical response in the form of multiplasmon absorption, with exciting implications in classical and quantum nonlinear optics. Specifically, we predict that graphene nanoribbons can be used as saturable absorbers with low saturation intensity in the farinfrared and terahertz spectrum. Moreover, we predict that two-plasmon absorption and extreme localization of plasmon fields in graphene nanodisks can lead to a plasmon blockade effect, in which a single quantized plasmon strongly suppresses the possibility of exciting a second plasmon.
\end{abstract}

DOI: 10.1103/PhysRevLett.114.236801

PACS numbers: 73.20.Mf, 42.50.Hz, 78.67.Wj, 79.20.Ws

The field of nonlinear optics ranges from fundamental questions concerning light-matter interactions to exciting technological applications [1]. However, usually very large field intensities are required to observe nonlinear effects. One is thus always looking for systems that will exhibit nonlinear phenomena at lower powers, with the ultimate limit being strong interactions between just two quanta of light [2]. One possibility to increase nonlinear effects is to use the strong localization and enhancement of electromagnetic fields in the form of surface plasmon excitations [3]. In that regard, we note that graphene [4] has been demonstrated to support extremely localized plasmons [5-14]. While optical nonlinearities in graphene have been studied by several authors [15-26], here we predict a novel nonlinear effect in the form of multiplasmon absorption. We also show how this effect leads to saturable absorption in graphene nanoribbons at low input powers in the farinfrared and terahertz spectrum. Moreover, we predict that the extreme localization of plasmon fields in graphene nanodisks leads to such a strong two-plasmon absorption that it becomes nearly impossible to excite a second quantized plasmon in the system. This plasmon blockade effect would cause the nanodisk to behave essentially like a quantum two-level system, which is observable in its resonance fluorescence spectrum.

Graphene is a two-dimensional hexagonal lattice of carbon atoms [4]. The low-energy band structure of graphene is described by Dirac cones with the electron dispersion $E_{n \mathbf{k}}=n \hbar v_{F}|\mathbf{k}|$, where $v_{F}=10^{6} \mathrm{~m} / \mathrm{s}$ and $n= \pm 1$ stands for the conduction (valence) band [27]. In its intrinsic form graphene is a zero-gap semiconductor; however, it can also be easily doped with free carriers and as such it supports plasmon modes [5-7]. At low frequencies, one can get a rather accurate description of these modes by using a simple Drude conductivity

$$
\sigma(\omega)=\frac{e^{2} E_{F}}{\pi \hbar^{2}} \frac{i}{\omega+i \gamma},
$$

where $E_{F}$ is the Fermi energy of graphene and $\gamma$ is a phenomenological damping rate that takes into account various decay channels like impurity or phonon scattering [7]. The resulting plasmon dispersion is given by

$$
q=\frac{2 \pi \varepsilon_{0} \bar{\varepsilon}_{r} \hbar^{2}}{e^{2} E_{F}} \omega^{2}
$$

and we assume the average dielectric permittivity $\bar{\varepsilon}_{r} \approx 2.5$, which roughly corresponds to the case of graphene on a $\mathrm{SiO}_{2}$ substrate and air on top. This simple Drude model breaks down at large frequencies when plasmons become strongly damped by electron-hole excitations, which can be described by the random phase approximation [5-7]. However, at low energies the Pauli principle blocks this decay channel and the plasmon is a long-lived excitation [5-14]. The resulting plasmon wavelength $\lambda_{p}=2 \pi / q_{p}$ is about 100 times smaller than the free space wavelength $\lambda=2 \pi c / \omega$, leading to the extreme localization of electromagnetic fields [7].

An intuitive explanation of the strong nonlinearities associated with plasmons emerges by considering the typical doping levels in graphene. For an electron density of $n=10^{12} \mathrm{~cm}^{-2}$, the distance between two electrons is $r_{e}=1 / \sqrt{n \pi}=5.6 \mathrm{~nm}$, and so to observe some kind of nonlinear phenomenon we need to compete with an intrinsic electric field of the order

$$
E_{e}=\frac{e}{4 \pi \bar{\varepsilon}_{r} \varepsilon_{0} r_{e}^{2}} \approx 2 \times 10^{7} \mathrm{~V} / \mathrm{m}
$$

This is significantly smaller than the characteristic field amplitude associated with nonlinear effects in atoms [1], given by the field between an electron and proton at a distance of a Bohr radius $a_{B}=0.5 \AA$. The electric field in that case is $E_{\text {at }}=5 \times 10^{11} \mathrm{~V} / \mathrm{m}$, about 4 orders of magnitude larger than the field $E_{e}$ in graphene.

To see what happens to plasmons at such a field strength $E_{e}$, let us imagine a general case of plasma oscillations at 
frequency $\omega$ and wave vector $q$, which is accompanied by an electric field $E(\mathbf{r}, t)=\frac{1}{2} E_{p} e^{i \mathbf{q} \cdot \mathbf{r}-i \omega t}+$ c.c. in the plane of graphene. When the plasmon field is small, it will induce a surface charge density $\rho_{p}=[q \sigma(\omega) / \omega] E_{p}$ that in turn creates an electric field $E_{p}=-i \rho_{p} / 2 \bar{\varepsilon}_{r} \varepsilon_{0}$, thus driving self-sustained charge density oscillations. However, at the field strength $E_{p}=E_{e}$, the induced charge density $\left|\rho_{p}\right|=$ en $/ 2$ will be comparable to the initial charge density en, and this simple picture of plasmons breaks down. We will in fact see that at this field $E_{e}$ there is a strong plasmon damping via multiplasmon absorption.

To understand how this comes about, let us first look at the linear (single-plasmon) absorption. Assuming that the graphene plane is perpendicular to the $z$ axis, the plasmon field can be described by the scalar potential $\varphi(\mathbf{r}, z, t)=\frac{1}{2} \varphi_{p} e^{i \boldsymbol{q} \cdot \mathbf{r}-q|z|} e^{-i \omega t}+$ c.c., $\quad$ where $\quad \mathbf{r}=(x, y)$. The electric field is then given by $\mathbf{E}=-\nabla \varphi$, with the amplitude $\left|E_{p}\right|=q\left|\varphi_{p}\right|$, for both in-plane and out-of-plane components. The interaction of the plasmon field with the electrons can be described by the Hamiltonian $H_{p}=-\frac{1}{2} e \varphi_{p} \sum_{j} e^{i \mathbf{q} \cdot \hat{\mathbf{r}}_{j}} e^{-i \omega t}+$ c.c., where $\hat{\mathbf{r}}_{j}$ is the position operator acting on the $j$ th electron. To calculate the linear absorption we can write the dissipated power as $P=\sum_{n} \hbar \omega\left[d w_{n 0}^{(1)} / d t\right]$, where

$$
\frac{d w_{n 0}^{(1)}}{d t}=\frac{2 \pi}{\hbar}\left|\left\langle n\left|H_{p}\right| 0\right\rangle\right|^{2} \delta\left(E_{n}-E_{0}-\hbar \omega\right)
$$

is Fermi's "golden rule" for the probability that the system will absorb one plasmon quantum of energy $\hbar \omega$. Here $|n\rangle$ is the many-body excited state of momentum $\hbar \mathbf{q}$ and energy $\hbar \omega$, with respect to the ground state $|0\rangle$, and we assume that the system is at zero temperature [28]. To quantify absorption we can look at the dissipation rate

$$
\gamma_{p}=\frac{P}{W} \text {. }
$$

Here $W=\bar{\varepsilon}_{r} \varepsilon_{0}\left|E_{p}\right|^{2} A / q$ is the total plasmon energy in the graphene flake of the surface area $A$ [28], while the figure of merit is the plasmon quality factor $Q=\omega / \gamma_{p}$.

From Eq. (4) we see that the absorption process consists of a sum over all events where the plasmon can excite a single $e-h$ pair. Conservation of energy and momentum require that $\hbar \omega=E_{n_{2} \mathbf{k}+\mathbf{q}}-E_{n_{1} \mathbf{k}}$; however, the Pauli principle allows this process only above the threshold condition of $\hbar \omega>2 E_{F}-\hbar v_{F} q$ [see the gray area in Fig. 1(a)]. We can calculate the linear absorption by using the basis of Dirac electron wave functions in graphene [28]. As an example, at energy $\hbar \omega=1.7 E_{F}$, the dissipation rate $\gamma_{p}^{(1)} / \omega=1$ is so high $(Q=1)$ that the plasmon is not a well-defined excitation. Below this threshold the Pauli principle blocks the absorption process and the plasmon is a long-lived quasiparticle. However, if we increase the plasmon field, higher-order (nonlinear) absorption must be accounted for as well.
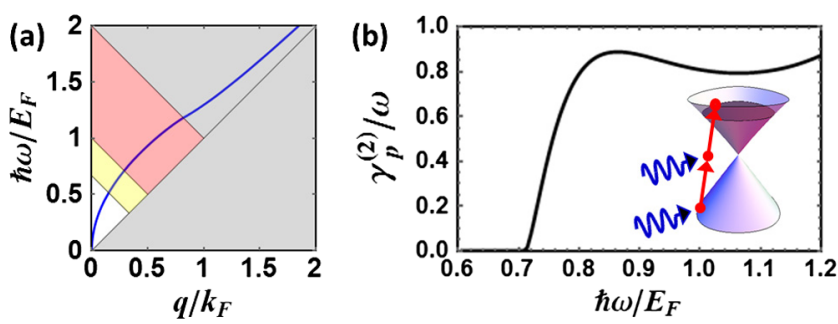

FIG. 1 (color online). (a) Plasmon dispersion relation. Gray area denotes the regime where a single plasmon can excite an $e-h$ pair. Red area denotes the regime where this process is forbidden but plasmons can decay via two-plasmon absorption. Similarly, the yellow area denotes the regime of three-plasmon absorption. (b) Two-plasmon damping rate $\gamma_{p}^{(2)} / \omega=F^{(2)}(\omega)$ for $E_{p}=E_{e}$, indicating that plasmons cannot oscillate when the plasmon field is equal to the intrinsic electric field. Inset shows the band structure of graphene and a two-plasmon absorption process.

We note that this simple calculation of the linear absorption gives the same result as the random phase approximation [28]. Encouraged by this fact, we proceed to calculate the nonlinear, two-plasmon absorption by writing $P=\sum_{n} 2 \hbar \omega\left[d w_{n 0}^{(2)} / d t\right]$ and using Fermi's golden rule for the probability that the system absorbs two plasmon quanta:

$\frac{d w_{n 0}^{(2)}}{d t}=\frac{2 \pi}{\hbar}\left|\sum_{m} \frac{\left\langle n\left|H_{p}\right| m\right\rangle\left\langle m\left|H_{p}\right| 0\right\rangle}{E_{m}-E_{0}-\hbar \omega}\right|^{2} \delta\left(E_{n}-E_{0}-2 \hbar \omega\right)$.

Alternatively, one could perform a third order expansion of the single particle density matrix including the screening fields consistently in every order of the expansion [34]. Such a calculation yields additional terms that contribute to the dissipation, arising from higher-order screening correlations. However, at high fields, when the nonlinear absorption is large, the screening process will be less effective and the simple calculation (6) should give a good estimate of the absorption. By evaluating expression (6) we get the two-plasmon absorption rate

$$
\frac{\gamma_{p}^{(2)}}{\omega}=F^{(2)}(\omega)\left|\frac{E_{p}}{E_{e}}\right|^{2},
$$

where $F^{(2)}$ is a dimensionless function of plasmon frequency, which is given in the Supplemental Material [28] and shown in Fig. 1(b). It is straightforward to show that the Pauli principle allows two-plasmon absorption only above the threshold $\hbar \omega>E_{F}-\hbar v_{F} q$ [see the red area in Fig. 1(a)]. Then, if we look at the energy $\hbar \omega=E_{F}$, a single plasmon cannot excite an $e-h$ pair but it can decay via two-plasmon absorption. Specifically, at this energy and the plasmon field $E_{p}=E_{e}$, the two-plasmon absorption rate $\gamma_{p}^{(2)} / \omega=0.8$, so that $E_{p} \lesssim E_{e}$ sets a maximum field amplitude in which plasmons can oscillate. In fact, at this 
intrinsic field we expect that the perturbation theory should fall apart entirely. Indeed at the same energy $\hbar \omega=E_{F}$ and field strength $E_{p}=E_{e}$ we obtain the three-plasmon absorption rate $\gamma_{p}^{(3)} / \omega=0.3$ [28], which is about $40 \%$ of the two-plasmon absorption rate, signalling the breakdown of the perturbation theory. Moreover, at the same field strength but lower energy $\hbar \omega=0.6 E_{F}$ [in the yellow area in Fig. 1(a), where two-plasmon absorption is forbidden by the Pauli principle], we obtain the three-plasmon absorption rate $\gamma_{p}^{(3)} / \omega=3.2$, again showing that plasmons cannot oscillate at the intrinsic field $E_{e}$.

These effects could be observed in experiments as the broadening of the plasmon linewidth with increasing plasmon amplitude. One way to excite plasmons by far field radiation is to use the sharp metal tip [12-14]. While the metal tip also leads to field enhancement, this is not the best setup to observe nonlinear effects, since the plasmon amplitude decreases as the plasmon propagates away from the tip in the form of a circular wave. Probably the dominant nonlinear effect will be a lower tip-plasmon coupling strength at high pump fields due to larger plasmon linewidths just below the tip.

An alternative way to excite plasmons by far-field radiation is to use graphene nanostructures with dipolar resonances [9-11], described by the polarizability [35,36]

$$
\alpha(\omega)=D^{3} \frac{G}{\frac{K}{\bar{\varepsilon}_{r}}-\frac{4 \pi \varepsilon_{0} i \omega D}{\sigma(\omega)}} .
$$

Particularly, in the case of a nanoribbon, $K=16$ and $G=L / D$, where $D$ is the width and $L \gg D$ is the length of the ribbon [36]. To describe the plasmon resonance we can use the simple Drude model of the surface conductivity $\sigma(\omega)$ given by Eq. (1). However, now we must include the total damping rate $\gamma_{t}=\gamma+\gamma_{p}^{(N)}$, which contains both the linear term $(\gamma)$ like impurity or phonon scattering, and the nonlinear term $\left(\gamma_{p}^{(N)}\right)$ like two- or three-plasmon absorption [28]. Specifically, to produce a resonance at frequency $\omega_{0}$, we require a ribbon of width $D=$ $\left(K / 4 \pi^{2}\right) \lambda_{p} \approx \lambda_{p} / 2$, where $\lambda_{p}=2 \pi / q_{p}$ is the plasmon wavelength in extended graphene given by Eq. (2). We are primarily interested in the regime $\hbar \omega_{0}=E_{F}$ where a single plasmon cannot excite an $e$ - $h$ pair, but two-plasmon absorption is allowed. For a typical doping $n=10^{12} \mathrm{~cm}^{-2}$ $\left(E_{F}=0.12 \mathrm{eV}\right)$, the corresponding free-space wavelength is $\lambda_{0}=2 \pi c / \omega_{0}=10.6 \mu \mathrm{m}$, the plasmon wavelength $\lambda_{p}=62 \mathrm{~nm}$, and the ribbon width $D=25 \mathrm{~nm}$.

The absorption cross section is given by $\sigma_{a}(\omega)=$ $(4 \pi \omega / c) \operatorname{Im} \alpha(\omega)=(3 / 8 \pi) \lambda_{0}^{2} \gamma_{r} \gamma_{t} /\left[\left(\omega_{0}-\omega\right)^{2}+\gamma_{t}^{2} / 4\right]$, where we have introduced the radiative decay rate of the ribbon $\gamma_{r}=\left(16 \pi^{3} / 3\right)\left(\bar{\varepsilon}_{r} G / K\right)\left(D^{3} / \lambda_{0}^{3}\right) \omega_{0}[36,37]$. To estimate impurity or phonon scattering we can use measurements of the direct current mobility $\gamma=e v_{F} / \mu \hbar \sqrt{\pi n}$ [7]. For typical graphene mobilities of $\mu=10^{4} \mathrm{~cm}^{2} / \mathrm{V} \mathrm{s}$ [4], we have $\gamma=8.6 \mathrm{THz}$, while $\gamma_{r}=6.1 \mathrm{GHz}$, even for a ribbon of length $L=100 D$. Since $\gamma_{r} \ll \gamma$, graphene nanostructures primarily act as absorbers, while the absorbed power can easily be detected via the reduction of light transmitted across the ribbon [9-11] [see Fig. 2(a)].

The dissipated power is $P=I \sigma_{a}=\gamma_{t} W$, where $W$ is the total energy, and $I=\frac{1}{2} \varepsilon_{0} c|E|^{2}$ is the incident light intensity. We can then estimate the plasmon field inside of the ribbon by using the result for the extended graphene $W=$ $\bar{\varepsilon}_{r} \varepsilon_{0}\left|E_{p}\right|^{2} A / q_{p}$, where $A=L D$ is the ribbon area. We obtain $\left|E_{p}\right|^{2}=|E|^{2} \omega_{0}^{2} / 4\left[\left(\omega_{0}-\omega\right)^{2}+\gamma_{t}^{2} / 4\right]$, where the total damping rate $\gamma_{t}=\gamma+\gamma_{p}^{(2)}$ itself depends on the plasmon field through the nonlinear damping term $\gamma_{p}^{(2)} \propto\left|E_{p}\right|^{2}$ given by Eq. (7). Then, by increasing the intensity, there is an increase in the total damping rate and a decrease of the absorption cross section [see Fig. 2(b)]. In particular, at intensity $I_{s}=24 \mathrm{~kW} / \mathrm{cm}^{2}$, we obtain $\gamma_{p}^{(2)}=\gamma$ on resonance, which reduces the absorption cross section by a factor of 2 . This corresponds to an input power of only $7 \mathrm{~mW}$ for a laser focused to a diffraction-limited spot size $(\lambda / 2)^{2}$, which would induce negligible heating of the graphene flake [28].

While extended graphene can also be used as a saturable absorber, due to Pauli blocking, the required intensities $\left(300 \mathrm{MW} / \mathrm{cm}^{2}\right)$ are 4 orders of magnitude higher [18]. Moreover, by lowering the doping we can further reduce the saturation intensity of the ribbon, while the resonance frequency would fall into the terahertz band, which would also require wider ribbons to satisfy the condition $\hbar \omega_{0}=E_{F}$. On the other hand, for a fixed ribbon width, by changing the doping one can tune the system response from the single-plasmon, two-plasmon or three-plasmon absorption regime. However, even though plasmon resonances in graphene nanoribons have been measured in the recent experiment [11], due to higher doping and lower quality factors, the saturation intensity required to see multiplasmon absorption is $I_{s} \approx 100 \mathrm{MW} / \mathrm{cm}^{2}$. This intensity would actually damage the graphene under the
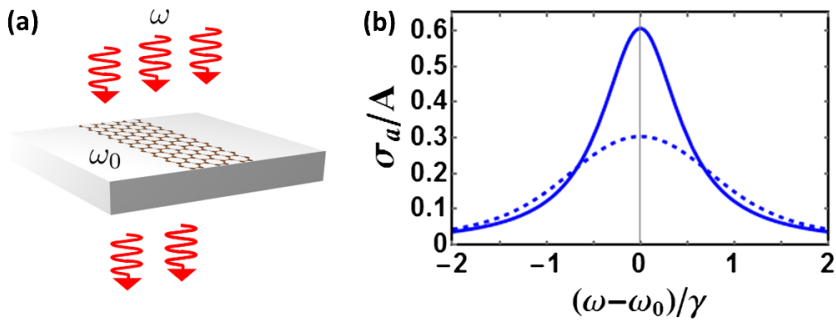

FIG. 2 (color online). (a) Transmission spectroscopy of a graphene nanoribbon. (b) Absorption cross section normalized to the surface area of the ribbon. The solid line stands for the low pump $I \ll I_{s}$, and the dashed line for the high pump intensity $I_{s}=24 \mathrm{~kW} / \mathrm{cm}^{2}$. The saturation of absorption is caused by twoplasmon absorption inside of the ribbon. The ribbon width is $D=25 \mathrm{~nm}$, the doping $n=10^{12} \mathrm{~cm}^{-2}$, and the resonance frequency $\omega_{0}=2 \pi \times 28 \mathrm{THz}$. 
continuous wave pump [28], which is the regime used in the experiment [11].

Especially interesting is the case of a nanodisk where we can localize the entire field to an extremely small volume $\approx \lambda_{p}^{3}$. The polarizability of a disk can also be described by expression (8), if we now take $D$ as the disk diameter, $K=12.5$, and $G=0.65$ [35]. To produce a resonance at energy $\hbar \omega_{0}=E_{F}$ and doping $n=10^{12} \mathrm{~cm}^{-2}$ requires a disk diameter $D=20 \mathrm{~nm}$. This yields a radiative decay rate of $\gamma_{r}=24 \mathrm{MHz}$, while the other parameters are the same as in the case of a ribbon (above).

We can now estimate the electric field amplitude associated with a single quantized plasmon by writing $W=\hbar \omega_{0}$ and using the result for the extended graphene $W=\bar{\varepsilon}_{r} \varepsilon_{0}\left|E_{p}\right|^{2} A / q_{p}$, where $A=\pi D^{2} / 4$ is the disk area. This gives a remarkable result for the field amplitude

$$
E_{p}^{Q}=\sqrt{\frac{2^{7} \pi^{4}}{K^{2}} \frac{\hbar \omega_{0}}{\varepsilon_{0} \bar{\varepsilon}_{r} \lambda_{p}^{3}}} \approx 2 \times 10^{7} \mathrm{~V} / \mathrm{m} .
$$

In other words, the field of a single quantized plasmon is of the same order of magnitude as the intrinsic field $E_{e}$. Then, for two plasmons the damping rate due to two-plasmon absorption would be so high $\left(\gamma_{p}^{(2)} / \omega_{0}=1.4\right)$ that the resonance peak would completely disappear, leading to a plasmon blockade effect [28].

To quantify this effect we adopt a density matrix approach, $d \rho / d t=(i / \hbar)[\rho, H]+\mathcal{L}[\rho]$. Here, the system Hamiltonian is given by $H=\hbar \omega_{0} N-(\hbar \Omega / 2)\left(a e^{i \omega t}+\right.$ $\left.a^{+} e^{-i \omega t}\right)$, where $N=a^{+} a$ is the plasmon number operator and $\Omega=\sqrt{(3 / 2 \pi)\left(\lambda_{0}^{2} I / \hbar \omega_{0}\right) \gamma_{r}}$ is the Rabi frequency describing the interaction with the incident field of intensity I. The Liouvillian $\mathcal{L}=\mathcal{L}^{(1)}+\mathcal{L}^{(2)}$ consists of two terms that characterize the linear and nonlinear dissipation, respectively; $\quad \mathcal{L}^{(N)}[\rho]=\left(\gamma^{(N)} / 2\right)\left(2 a^{N} \rho a^{+N}-a^{+N} a^{N} \rho-\right.$ $\rho a^{+N} a^{N}$ ) [38], where $\gamma^{(1)}=\gamma$ and $\gamma^{(2)}=\gamma_{p}^{(2)}$, so that $\gamma^{(1)} / \gamma^{(2)}=0.03 \ll 1$. Under conditions of weak external driving, the dynamics of the (infinite-dimensional) density matrix can effectively be truncated to a few-excitation manifold [38]. Specifically, at $\Omega \ll \gamma^{(2)}$ the steady-state population of the excited state $|2\rangle$ (containing two plasmons) is extremely weakly populated, $\rho_{22}=2 \rho_{11} /[1+$ $\left.\left(2 \gamma^{(2)} / \Omega\right)^{2}\right]$, and the disk effectively behaves as a twolevel system.

The absorption cross section $\sigma_{a}=\left(\hbar \omega_{0} \Omega / I\right) \operatorname{Im} \rho_{10}=$ $(3 / 8 \pi) \lambda_{0}^{2} \gamma_{r} \gamma /\left[\left(\omega_{0}-\omega\right)^{2}+\gamma^{2} / 4+\Omega^{2} / 2\right]$ saturates at intensity $I_{s}=50 \mathrm{~kW} / \mathrm{cm}^{2}(\Omega=\gamma / \sqrt{2})$. Like in the ribbon case, the radiative damping rate is negligible $\left(\gamma_{r} \ll \gamma\right)$ and the disk primarily acts as an absorber. However, the weakly scattered light will now show interesting spectral properties since a two-level system behaves as a strong frequency mixer [38]. To substantiate this, let us look at the power spectrum of the scattered light, $S\left(\omega_{s}\right)=$ $(1 / \pi) \operatorname{Re} \int_{0}^{\infty}\left\langle a^{+}(0) a(t)\right\rangle e^{i \omega_{s} t} d t$. By using the quantum regression theorem [38] we obtain the steady-state two-time correlation function on resonance, $\left\langle a^{+}(0) a(t)\right\rangle=$ $\frac{1}{4}\left(e^{-\gamma t / 2}+\frac{1}{2} e^{-3 \gamma t / 4} e^{-i \Omega t}+\frac{1}{2} e^{-3 \gamma t / 4} e^{i \Omega t}\right) e^{-i \omega t}$, where we have assumed the strong-pump regime $\Omega \gg \gamma / 4$ but also $\Omega \ll \gamma^{(2)}$ so that the disk still behaves as a two-level system. The scattered spectrum consists of the Mollow triplet: one peak at the laser frequency $\omega_{0}$ and two at the Rabi sidebands $\omega_{0} \pm \Omega$ [39]. In Fig. 3(d) we plot the case of $\Omega=4 \gamma$, while $\Omega / \gamma^{(2)}=0.1$ so that the disk still behaves as a two-level system up to an excellent approximation. A peculiar feature of this result is that the system produces frequency mixing (characteristic of dispersive nonlinearities) starting only from the two-plasmon absorptive nonlinearity.

The two-level nature of the system is especially reflected in the second-order correlation function $g^{(2)}(t)=$ $\left\langle a^{+}(0) a^{+}(t) a(t) a(0)\right\rangle /\left\langle a^{+}(0) a(0)\right\rangle^{2}$, which describes the probability of detecting a second scattered photon at time $t$ given a detection event at $t_{0}=0$. In the steady-state, low pump regime $\Omega \ll \gamma$, it is straightforward to show that $g^{(2)}(0)=\left(1+\gamma^{(2)} / \gamma\right)^{-2}=10^{-3}$. Thus, the disk exhibits an almost perfect antibunching effect characteristic of an ideal two-level system [to compare, $g^{(2)}(0)=1$ in the absence of nonlinearities]. The temporal duration of this antibunching dip around zero time delay $t=0$ is given by $\sim 1 / \gamma$ [in particular, within a two-level approximation $g^{(2)}(t)=1+$ $e^{-\gamma t}-2 e^{-\gamma t / 2}$ [38]], after which the system returns to a stationary value of $g^{(2)}(t)=1$ as illustrated in Fig. 3(e). The sub-Poissonian nature of the scattered light, $g^{(2)}(0)<g^{(2)}(t)$, is a distinctly nonclassical feature that

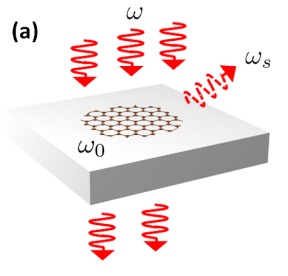

(d) 4

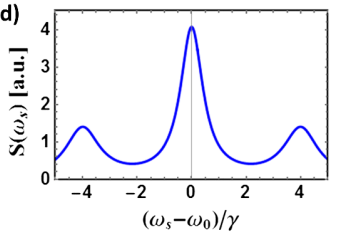

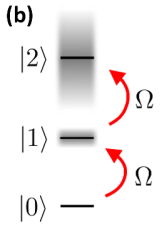
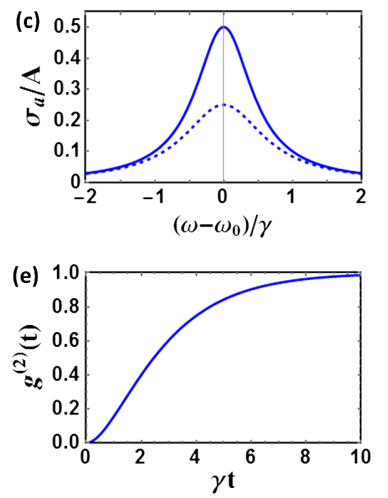

FIG. 3 (color online). (a) Resonance fluorescence of a graphene nanodisk. (b) Quantized plasmon energy levels in a disk. Twoplasmon absorption induces a huge linewidth of the doubly excited state $|2\rangle$, which makes it extremely difficult to populate this state and effectively turns the disk into a two-level system. (c) Absorption cross section normalized to the disk surface. The solid line stands for the low pump field $(\Omega \ll \gamma)$, and the dashed line for the strong pump field $(\Omega=\gamma / \sqrt{2})$. (d) Scattered spectrum on resonance $\left(\omega=\omega_{0}\right)$ in the strong pump regime $(\Omega=4 \gamma)$, showing the Mollow triplet. (e) The second-order correlation function $g^{(2)}(t)$ of the scattered field for weak pump intensities, showing an antibunching effect $g^{(2)}(0) \ll 1$ characteristic of a two-level system. 
reflects the inability of a two-level system to emit two excitations simultaneously [40].

In conclusion, we have discussed multiplasmon absorption in graphene, and we have shown how this effect leads to the saturation of absorption in graphene nanoribbons, which could be used to build tunable low-power saturable absorbers in the infrared and terahertz band [18]. We have also shown that two-plasmon absorption in graphene nanodisks leads to the plasmon blockade effect, which offers an exciting possibility to observe and understand strongly interacting photons at the many-body quantum level [41]. Multiplasmon absorption could also have interesting effects on the hot-carrier dynamics [42] and graphene photodetection or light harvesting devices [43]. Finally, we expect that exciting new physics could be found beyond the perturbative regime discussed in our Letter, like high-harmonic generation observed at strong light intensities [44,45].

The authors would like to acknowledge the support of the European Commission and the Croatian Ministry of Science, Education and Sports (Co-Financing Agreement No. 291823, Marie Curie FP7-PEOPLE-2011-COFUNDNEWFELPRO-GRANQO), and European Commission (FP7-ICT-2013-613024-GRASP).

*mjablan@phy.hr

[1] R. W. Boyd, Nonlinear Optics (Academic Press, Amsterdam, 2008).

[2] D. E. Chang, V. Vuletić, and M. D. Lukin, "Quantum nonlinear optics-photon by photon," Nat. Photonics 8, 685 (2014).

[3] M. Kauranen and A. V. Zayats, "Nonlinear plasmonics," Nat. Photonics 6, 737 (2012).

[4] K. S. Novoselov, A. K. Geim, S. V. Morozov, D. Jiang, Y. Zhang, S. V. Dubonos, I. V. Grigorieva, and A. A. Firsov, "Electric field effect in atomically thin carbon films," Science 306, 666 (2004).

[5] B. Wunsch, T. Stauber, F. Sols, and F. Guinea, "Dynamical polarization of graphene at finite doping," New J. Phys. 8, 318 (2006).

[6] E.H. Hwang and S. Das Sarma, "Dielectric function, screening, and plasmons in two-dimensional graphene," Phys. Rev. B 75, 205418 (2007).

[7] M. Jablan, H. Buljan, and M. Soljačić, "Plasmonics in graphene at infrared frequencies," Phys. Rev. B 80, 245435 (2009).

[8] M. Jablan, M. Soljačić, and H. Buljan, "Plasmons in graphene: Fundamental properties and potential applications," Proc. IEEE 101, 1689 (2013).

[9] L. Ju, B. Geng, J. Horng, C. Girit, M. Martin, Z. Hao, H. A. Bechtel, X. Liang, A. Zettl, Y. R. Shen, and F. Wang, "Graphene plasmonics for tunable terahertz metamaterials," Nat. Nanotechnol. 6, 630 (2011).

[10] H. Yan, X. Li, B. Chandra, G. Tulevski, Y. Wu, M. Freitag, W. Zhu, P. Avouris, and F. Xia, "Tunable infrared plasmonic devices using graphene/insulator stacks," Nat. Nanotechnol. 7, 330 (2012).

[11] H. Yan, T. Low, W. Zhu, Y. Wu, M. Freitag, X. Li, F. Guinea, P. Avouris, and F. Xia, "Damping pathways of mid-infrared plasmons in graphene nanostructures," Nat. Photonics 7, 394 (2013).

[12] J. Chen, M. Badioli, P. Alonso-Gonzalez, S. Thongrattanasiri, F. Huth, J. Osmond, M. Spasenović, A. Centeno, A. Pesquera, P. Godignon, A. Z. Elorza, N. Camara, F. J. Garcia de Abajo, R. Hillenbrand, and F. H. L. Koppens, "Optical nanoimaging of gate-tuneable graphene plasmons," Nature (London) 487, 77 (2012).

[13] Z. Fei, A. S. Rodin, G. O. Andreev, W. Bao, A. S. McLeod, M. Wagner, L. M. Zhang, Z. Zhao, M. Thiemens, G. Dominguez, M. M. Fogler, A. H. Castro Neto, C. N. Lau, F. Keilmann, and D. N. Basov, "Gate-tuning of graphene plasmons revealed by infrared nanoimaging," Nature (London) 487, 82 (2012).

[14] A. Woessner, M. B. Lundeberg, Y. Gao, A. Principi, P. Alonso-Gonzalez, M. Carrega, K. Watanabe, T. Taniguchi, G. Vignale, M. Polini, J. Hone, R. Hillenbrand, and F. H. L. Koppens, "Highly confined low-loss plasmons in graphene-boron nitride heterostructure," Nat. Mater. 14, 421 (2015).

[15] S. A. Mikhailov and K. Zeigler, "Nonlinear electromagnetic response of graphene: frequency multiplication and the selfconsistent-field effects," J. Phys. Condens. Matter 20, 384204 (2008).

[16] A. R. Wright, X. G. Xu, J. C. Cao, and C. Zhang, "Strong nonlinear optical response of graphene in the terahertz regime," Appl. Phys. Lett. 95, 072101 (2009).

[17] K. L. Ishikawa, "Nonlinear optical response of graphene in time domain,"Phys. Rev. B 82, 201402(R) (2010).

[18] Z. Sun, T. Hasan, F. Torrisi, D. Popa, G. Privitera, F. Wang, F. Bonaccorso, D. M. Basko, and A. C. Ferrari, "Graphene mode-locked ultrafast laser," ACS Nano 4, 803 (2010).

[19] E. Hendry, P. J. Hale, J. Moger, A. K. Savchenko, and S. A. Mikhailov, "Coherent Nonlinear Optical Response of Graphene," Phys. Rev. Lett. 105, 097401 (2010).

[20] S. A. Mikhailov, "Theory of the giant plasmon-enhanced second-harmonic generation in graphene and semiconductor two-dimensional electron systems," Phys. Rev. B 84, 045432 (2011).

[21] H. Yang, X. Feng, Q. Wang, H. Huang, W. Chen, A. T. S. Wee, and W. Ji, "Giant two-photon absorption in bilayer graphene," Nano Lett. 11, 2622 (2011).

[22] X. Yao and A. Belyanin, "Giant Optical Nonlinearity of Graphene in a Strong Magnetic Field," Phys. Rev. Lett. 108, 255503 (2012).

[23] M. Gullans, D. E. Chang, F. H. L. Koppens, F. J. Garcia de Abajo, and M. D. Lukin, "Single-Photon Nonlinear Optics with Graphene Plasmons," Phys. Rev. Lett. 111, 247401 (2013).

[24] M. T. Manzoni, I. Silvero, F. J. Garcia de Abajo, and D. E. Chang, "Second-order quantum nonlinear optical processes in graphene nanostructures," arXiv:1406.4360v1.

[25] J. D. Cox and F. J. Garcia de Abajo, "Electrically tunable nonlinear plasmonics in graphene nanoislands," Nat. Commun. 5, 5725 (2014). 
[26] I. Al-Naib, J. E. Sipe, and M. M. Dignam, "High harmonic generation in undoped graphene: Interplay of interand intraband dynamics," Phys. Rev. B 90, 245423 (2014).

[27] P. R. Wallace, “The band theory of graphite," Phys. Rev. 71, 622 (1947).

[28] See Supplemental Material at http://link.aps.org/ supplemental/10.1103/PhysRevLett.114.236801 for discussion and calculation of the plasmon energy, multiplasmon absorption rate, dispersive nonlinearity, single-plasmon nonlinearity, and finite temperature effects, which includes Refs. [29-33].

[29] N. W. Ahscroft and N. D. Mermin, Solid State Physics (Harcourt, Fort Worth, 1976).

[30] J. D. Jackson, Classical Electrodynamics (John Wiley \& Sons, New York, 1999).

[31] D. Pines, The Theory of Quantum Liquids (Benjamin, New York, 1966).

[32] J. J. Sakurai, Modern Quantum Mechanics (Addison-Wesley Publishing Company, Reading, Massachusetts, 1994).

[33] M. Freitag, M. Steiner, Y. Martin, V. Perebeinos, Z. Chen, J. C. Tsang, and P. Avouris, "Energy dissipation in graphene field-effect transistors," Nano Lett. 9, 1883 (2009).

[34] H. Ehrenreich and M. H. Cohen, "Self-consistent field approach to the many-electron problem," Phys. Rev. 115, 786 (1959).

[35] Z. Fang, S. Thongrattanasiri, A. Schlather, Z. Liu, L. Ma, Y. Wang, P. M. Ajayan, P. Nordlander, N. J. Halas, and F. J. Garcia de Abajo, "Gated tunability and hybridization of localized plasmons in nanostructured graphene," ACS Nano 7, 2388 (2013).

[36] F. J. Garcia de Abajo, "Graphene plasmonics: Challenges and opportunities," ACS Photonics 1, 135 (2014).

[37] S. Thongrattanasiri, F. H. L. Koppens, and F. J. Garcia de Abajo, "Complete Optical Absorption in Periodically Patterned Graphene,” Phys. Rev. Lett. 108, 047401 (2012).

[38] M. O. Scully and M. S. Zubairy, Quantum Optics (University Press, Cambridge, 1997).

[39] B. R. Mollow, "Power spectrum of light scattered by twolevel system," Phys. Rev. 188, 1969 (1969).

[40] L. Mandel, "Non-classical states of the electromagnetic field," Phys. Scr. T12, 34 (1986).

[41] A. Tomadin and R. Fazio, "Many-body phenomena in QEDcavity arrays," J. Opt. Soc. Am. B 27, A130 (2010).

[42] K. J. Tielrooij, J. C. W. Song, S. A. Jensen, A. Centeno, A. Pesquera, A. Z. Elroza, M. Bonn, L. S. Levitov, and F. H. L. Koppens, "Photoexcitation cascade and multiple hot-carrier generation in graphene," Nat. Phys. 9, 248 (2013).

[43] F. H. L. Koppens, T. Muller, Ph. Avouris, A. C. Ferrari, M. S. Vitiello, and M. Polini, "Photodetectors based on graphene, other two-dimensional materials and hybrid systems," Nat. Nanotechnol. 9, 780 (2014).

[44] P. B. Corkum, "Plasma Perspective on Strong Field Multiphoton Ionization," Phys. Rev. Lett. 71, 1994 (1993).

[45] M. Lewenstein, Ph. Balcou, M. Yu. Ivanov, A. L'Hullier, and P. B. Corkum, "Theory of high-harmonic generation by low-frequency laser fields," Phys. Rev. A 49, 2117 (1994). 